\title{
Vibrational excitons, resonant energy transfer, and local structure in liquid benzene*
}

\author{
R. LeSar ${ }^{\dagger}$ and R. Kopelman
}

Department of Chemistry. The University of Michigan, Ann Arbor, Michigan 48109

(Received 16 December 1976)

\begin{abstract}
The presence of vibrational excitons in liquid benzene has been tested by the method of isotopic dilution. A $_{6} \mathrm{H}_{6} / \mathrm{C}_{6} \mathrm{D}_{6}$ concentration study on the infrared and Raman fundamental modes reveals that the umbrella $\left(A_{2 u}\right)$ vibrational exciton in solid benzene retains its basic identity upon melting and at room temperature. The total liquid exciton bandwidth is about $40 \mathrm{~cm}^{-1}$, practically the same as in the solid. This indicates an instantaneous local liquid structure similar to that of the solid (the $\mathbf{C}_{i}$ crystal site symmetry is also nearly preserved), in general agreement with indications from other methods. The fastest nearest neighbor vibrational resonant transfer takes about 1 psec. The residual linewidth at isotopic dilution is $3-4 \mathrm{~cm}^{-1}$, which is due to inhomogeneous and/or homogeneous broadening. The respective overall reorientational and/or translational relaxation takes about $2 \mathrm{psec}$ or longer. The exciton linewidth is proportional to the square root of the isotopic concentration except for a sudden break at some critical concentration.
\end{abstract}

\section{INTRODUCTION}

Liquid benzene has been a test case for studying "local structure." Attempts to study such "local order" or "residual structure" have been made with a variety of techniques, including $\mathbf{x}$-ray diffraction by Narten ${ }^{1}$ and earlier investigators, ${ }^{2-4}$ sound velocity measurements, ${ }^{5}$ light scattering and depolarization, ${ }^{6-9}$ Raman (phonon) spectra, ${ }^{10,11} \mathrm{NMR},{ }^{12}$ and (at least by implication) dielectric relaxation and far-infrared spectra. ${ }^{13}$ We add here what we believe to be a very sensitive new technique - the vibrational exciton spectra.

Liquid benzene has also been a test case for relaxation studies, involving vibrational and reorientational relaxation, utilizing NMR, ${ }^{14} \mathrm{NQR},{ }^{15}$ Rayleigh scattering $^{16,17}$ and Raman spectra. ${ }^{18-20}$ We add here some data from infrared and Raman spectra.

Resonant energy transfer and excitons in liquids are of major interest. One of us proposed ${ }^{21}$ the existence of vibrational excitons in neat benzene liquid, based on a preliminary infrared study utilizing the isotopic dilution method for the confirmation of exciton assignments, as used in crystals. ${ }^{21-23}$ Since then a number of studies on resonant energy transfer, involving isotopic benzene solution s $^{20}$ as well as chemical benzene solutions, ${ }^{24}$ have appeared, as well as similar studies on other neat and isotopic dilute liquids. ${ }^{25}$ Theoretical studies on molecular excitons in liquids, ${ }^{26-28}$ experimental studies on trapped Wannier excitons in liquids, ${ }^{29}$ as well as many (implicit and explicit) studies on electronic exciton hopping in molecular liquids ${ }^{30-33}$ are also available.

In our present study we show that the vibrational exciton interactions, which have been demonstrated in crystal benzene studies to be of an extremely shortrange nature, ${ }^{22,34-37}$ are very sensitive to the immediate environment of a given molecule in the liquid. As the motion (exchange) of some of these excitons is fast compared to the other molecular relaxation processes in the liquid, these excitons map out the liquid structure over a "microspace" and "microtime" interval. Our results are in agreement with the best available experimental $^{1}$ and theoretical ${ }^{38}$ studies of the benzene liquid structure. In particular, we show that while most vi- brational excitons in solid benzene lose their exciton character upon melting, at least one survives this phase transition with only minor perturbations. This is the umbrella $\left(A_{2 u}\right)$ mode exciton. The respective resonant energy transfer rate is about 1 psec. Also, the local (instantaneous) liquid structure is quite similar to that of the solid.

\section{EXPERIMENTAL}

Isotopic mixtures of benzene and perdeuterobenzene were prepared by a weighing method. The concentrations of some of the samples were checked out by mass spectrometry. The benzene was thiophene-free analytical reagent grade from Mallincrodt Chemical Works, and the perdeuterobenzene was 99.5 at. $\%$ pure $\mathrm{C}_{\theta} \mathrm{D}_{6}$ obtained from Merck, Sharp and Dohme of Canada Ltd. Neither was further purified.

The infrared studies were carried out on a Beckman IR-12 with a resolution of about $1 \mathrm{~cm}^{-1}$ in the regions studied. The cell holder consisted of two copper rings with beveled edges standing out from the ring. The cell itself consisted of two KBr disks separated by a plastic spacer, with a sealing ring made of thin indium wire. The copper rings clamped down onto the indium wire. The liquid was inserted through a syringe (introduced through a cut in the plastic and sealed on the outside with indium). For the $A_{2 u}$ mode the sample thickness was about $15-20 \mu$, while for the other fundamentals it was greater (about $25 \mu$ for the pure liquid and $100 \mu$ for the dilute liquid).

The Raman spectra were obtained with a Coherent Radiation model CR-5 argon-ion laser and a Spex model 1401 double spectrometer using either photon counting or direct current detection. The $4965 \AA$ lasing line was used with the slits set in such a way that a bandpass of $2 \mathrm{~cm}^{-1}$ was obtained. The samples were sealed in a capillary. All this work was done at "room" temperature.

\section{RESULTS AND DISCUSSION}

Very often, the behavior and structure of liquids have been discussed in terms of a condensed gas model. We 


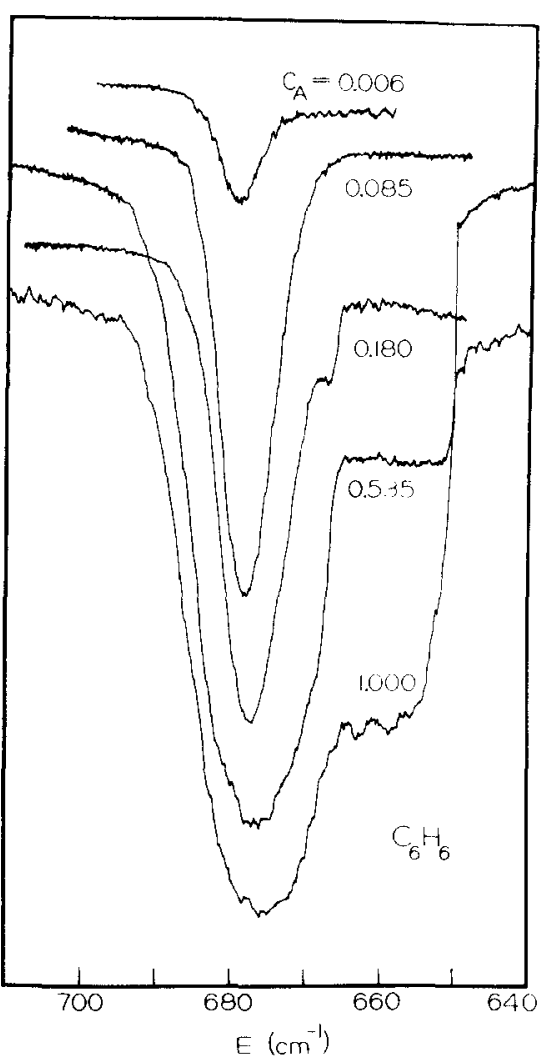

FIG. 1. $A_{2 u}$ vibrational mode for $\mathrm{C}_{6} \mathrm{H}_{6}$ in isotopic mixed liquids of $\mathrm{C}_{6} \mathrm{H}_{6}-\mathrm{C}_{6} \mathrm{D}_{6}$. The concentrations $\left(C_{A}\right)$ are given with respect to $\mathrm{C}_{6} \mathrm{H}_{6}$ (in mole fractions). Ambient ir spectra.

believe, however, that at least for molecular liquids like benzene, and near the melting point, it is better to discuss the structure of the liquid in terms of a "disordered solid" model.

In a molecular solid like benzene, vibrational excitons are of importance. These effects have been well studied for benzene, in particular for the $A_{2 u}$ vibrational mode. ${ }^{21,34,35}$ For a discussion on the development of cluster states in this mode see earlier papers from this group. ${ }^{36,37}$

While we don't expect exact long range symmetry in the liquid, on the short range, the relative positions of the molecules with respect to each other may be similar to those in the solid. If the vibrational exciton transfer is fast (on the order of $10^{-12}-10^{-13} \mathrm{sec}$ ), ${ }^{21,34}$ it is expected that these excitons will also be of prime importance in the liquid (especially when this "relaxation channel" is faster than all others).

In the crystal, the spectrum of a vibrational transition does not map out the vibrational exciton density of states. This is due to $\mathbf{k}$ selection rules, which are a consequence of the long range (translational) symmetry in the crystal. In a liquid, however, there is no long range symmetry and so in the spectrum of the liquid presumably the density of states of the levels involved in the transition is mapped out, as is the case for disordered solids. ${ }^{23}$ However, the width of the pure liquid spectrum does not just reflect the width of the vibrational exciton band. The exciton bandwidth, even if it dominates, is broadened by two factors: (1) homogeneous broadening due to molecular reorientational, translational, etc., relaxation; and (2) inhomogeneous broadening due to an inhomogeneous environment (these two effects are not completely separable).

If the pure liquid is isotopically diluted (such as benzene diluted with perdeuterobenzene), the exciton band will shrink as in the case of a solid (see, for instance, Fig. 2 of Ref. 39, where the CPA calculated exciton band for solid naphthalene is shown). Thus, if the vibrational exciton structure is a major contributor towards the bandwidth for a given transition, an appreciable shrinkage of the spectroscopic band should be observed upon dilution.

In Fig. 1, the spectra of the $A_{2 u}$ vibrational mode for liquid benzene are shown over a range of concentrations. As benzene $\left(\mathrm{C}_{6} \mathrm{H}_{6}\right)$ is diluted from $100 \%$ to $0.6 \%$, there is a sizeable decrease in the bandwidth corresponding to a shrinkage of the exciton band. This effect is better shown in Fig. 2, where the FWHH in $\mathrm{cm}^{-1}$ is plotted versus concentration. It can be seen that the FWHH is about $24 \mathrm{~cm}^{-1}$ at $100 \%$ and about 5.5 $\mathrm{cm}^{-1}$ at $0.6 \%$.

A similar, though not as marked, effect is shown in Fig. 3 for the perdeuterobenzene $A_{2 u}$ band, over a range of concentrations. The difference in the magnitudes of the effect will be discussed below.

In papers by Hoshen and Kopelman ${ }^{36}$ and LeSar and Kopelman, ${ }^{37}$ the cluster formation in benzene is discussed for the $A_{2 u}$ vibration in the solid. In the liquid, however, owing to the inhomogeneity of the environment of the molecules and/or motional broadening, no distinct clusters could be observed. However, as the concentration of benzene $\left(\mathrm{C}_{6} \mathrm{H}_{6}\right)$ is reduced to $1 \%$ or less, the molecules will be (instantaneously) in essentially "monomer" states. Therefore, the spectrum of a vibrational band of very dilute benzene will represent essentially a single vibrational state, "smeared out" by homogeneous and inhomogeneous broadening. Since the pure liquid spectrum represents the exciton band smeared out by the same above broadening effects, the difference in width of the pure liquid spectrum and the dilute spectrum should give a good approximation of the exciton bandwidth. In this case, using the differences in FWHH (in $\mathrm{cm}^{-1}$ ) and multiplying by 2 (assuming quasi-

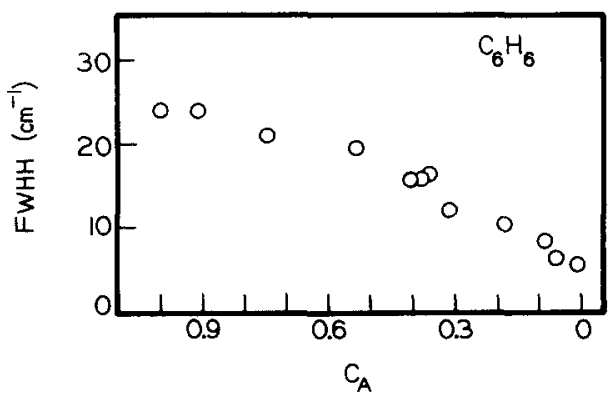

FIG. 2. FWHH vs concentration for the $A_{24}$ mode of $\mathrm{C}_{6} \mathrm{H}_{6}$ in mixed liquid $\mathrm{C}_{6} \mathrm{H}_{6}-\mathrm{C}_{6} \mathrm{D}_{6}$. The concentrations are the mole fractions of $\mathrm{C}_{6} \mathrm{H}_{6}$ in $\mathrm{C}_{6} \mathrm{D}_{6}$. From Fig. 1 spectra. 


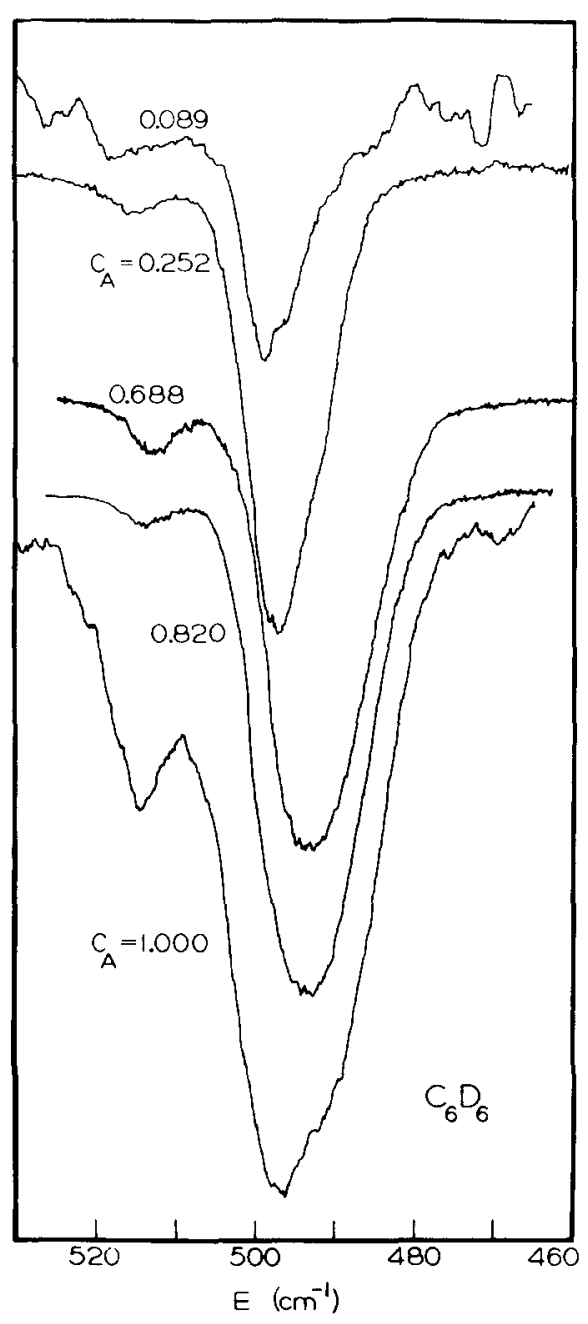

FIG. 3. $A_{24}$ vibrational mode for $\mathrm{C}_{6} \mathrm{D}_{6}$ in isotopic mixed liquids of $\mathrm{C}_{6} \mathrm{H}_{6}-\mathrm{C}_{6} \mathrm{D}_{6}$. The concentrations $\left(C_{A}\right)$ are given with respect to $\mathrm{C}_{6} \mathrm{D}_{6}$ (in mole fractions). Ambient ir spectra.

triangular band shapes), the width of the pure liquid exciton band is found to be about $37 \mathrm{~cm}^{-1}$.

In the crystal, the benzene and perdeuterobenzene $A_{2 u}$ vibrational modes fall into the "separated band limit. "23 It can be shown that the energy second moment $Y_{A}$ (which is approximately the FWHH in the absence of selection rules) is given by $Y_{A}=C_{A}^{1 / 2} Y_{0}$, where $C_{A}$ is the concentration and $Y_{0}$ the second moment of the pure

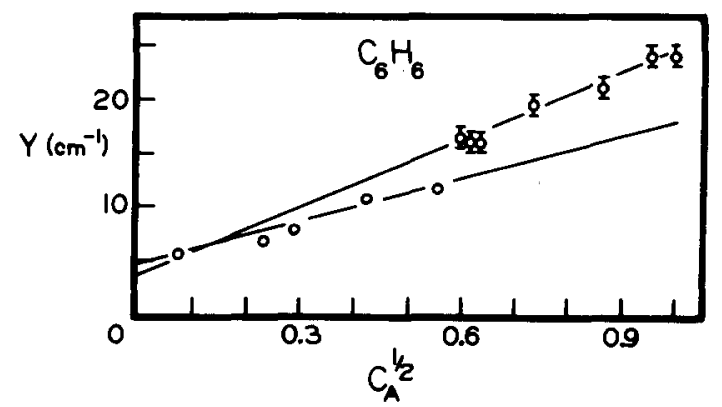

FIG. 4. FWHH $(Y)$ vs the square root of the concentration $C_{A}^{1 / 2}$ for the $A_{2 \mu}$ mode of $\mathrm{C}_{6} \mathrm{H}_{6}(A)$ in liquid solutions of $\mathrm{C}_{6} \mathrm{H}_{6}-\mathrm{C}_{6} \mathrm{D}_{6}$. Error bars are omitted where they approach the diameters of the circles.

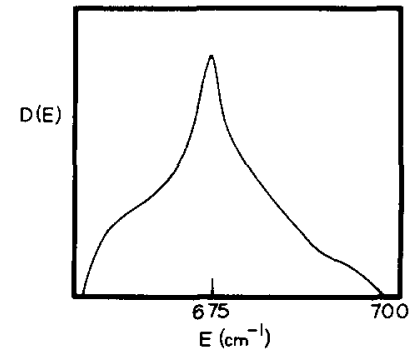

FIG. 5. Schematic density of states for pure liquid $\mathrm{C}_{6} \mathrm{H}_{6}$ (after Hoshen and Kopelman ${ }^{36,70}$ ).

sample. ${ }^{23}$ This relationship is not so useful in the crystal, as the residual $\mathbf{k}$ selection rules prohibit the entire band from appearing in the spectrum. ${ }^{23}$ In the liquid, however, one does see the entire band, so the equation should hold. In Fig. 4, the FWHH $(Y)$ is plotted versus $C_{A}^{1 / 2}$. As can be seen in this figure, there is a definite break into two separate lines at about $C_{A}=0.34$ (i.e., $\left.C_{A}^{1 / 2}=0.58\right)$. While it is difficult to quantitatively explain this break, a few possible explanations will be discussed below.

Both of the above lines extrapolate to about a $4.0 \mathrm{~cm}^{-1}$ width at zero concentration (4.4 for the lower line and 3. 3 for the upper line, the discrepancy being within experimental error). Using the value for the zero concentration width as $4.0 \mathrm{~cm}^{-1}$ and that for the pure liquid as $24 \mathrm{~cm}^{-1}$, this yields a result of $(2 \times 20=) 40 \mathrm{~cm}^{-1}$ for the width of the vibrational exciton density of states in the pure liquid. This result is a more accurate estimate than the previous $37 \mathrm{~cm}^{-1}$ one, since it is derived from the extrapolation to $C_{A}=0.0$.

The cause of the break in the curve of Fig. 4 into two lines is not well understood. There are two possible explanations. The first is that above $C_{A}=0.32$ the primary bandwidth is due to the exciton band but as the band shrinks the other broadening effects become important enough to cause a marked change in width. In other words, at about $34 \% \mathrm{C}_{6} \mathrm{H}_{6}$ in $\mathrm{C}_{6} \mathrm{D}_{6}$ one is going from one coupling case where the exciton effects dominate to another coupling case where the homogeneous and/or inhomogeneous broadening effects dominate. However, it is not then clear why the lower concentrations would also yield a linear relationship with $C_{A}^{1 / 2}$.

The $A_{2 u}$ vibrational exciton band of $\mathrm{C}_{6} \mathrm{H}_{8}$ is the broadest of all the crystal exciton bands in both benzene and perdeuterobenzene..$^{22,37}$ The bandwidths $(Y)$ in $\mathrm{cm}^{-1}$ for both the benzene and perdeuterobenzene infrared and Raman active fundamentals are shown in Table $I$ for both pure and dilute liquid samples. As can be seen, not even the degenerate $(E)$ modes have the same bandwidth as does the $A_{2 u}$ umbrella $\left(\nu_{11}\right)$ mode of $\mathrm{C}_{6} \mathrm{H}_{8}$. We note also the lessened width of the $\mathrm{C}_{6} \mathrm{D}_{6}$ umbrella $\left(A_{2 u}\right)$ mode. Hoshen and Kopelman ${ }^{36}$ calculated the vibrational exciton density of states for the pure benzene crystal and the bandwidth is shown to be up to $40 \mathrm{~cm}^{-1}$ (cf. Fig. 5). It has been shown by Laufer and Kopelman ${ }^{34}$ that the width of the exciton density of states is very dependent on the exciton pairwise interaction energies. Since the width of the exciton density of states in the liquid is so close to that in the crystal (they are the same within experimental error), it can be concluded that the ex- 
TABLE I. FWHH $\left(\mathrm{cm}^{-1}\right)$ for pure and dilute liquid benzene and perdeuterobenzene fundamentals (Raman, $\sim 2 \mathrm{~cm}^{-1}$ resolution).

\begin{tabular}{|c|c|c|c|c|c|c|c|c|c|c|c|}
\hline & $\nu_{1}\left(a_{1 s}\right)$ & $\nu_{2}\left(a_{1 g}\right)$ & $v_{6}\left(e_{2 g}\right)$ & $\nu_{7}\left(e_{2 g}\right)$ & $\nu_{8}\left(e_{2 g}\right)$ & $\nu_{9}\left(e_{2 g}\right)$ & $\nu_{10}\left(e_{2 g}\right)$ & $\nu_{1}+\nu_{6}\left(e_{2 g}\right)$ & $\nu_{11}\left(a_{2 u}\right)$ & $\nu_{18}\left(e_{1 u}\right)$ & $v_{19}\left(e_{1 u}\right)$ \\
\hline \multicolumn{12}{|c|}{$\overline{\mathrm{C}_{A}\left(\mathrm{C}_{6} \mathrm{H}_{6}\right)}$} \\
\hline 1.000 & $3.7^{\mathrm{a}}$ & 13.0 & $8.5^{\mathrm{a}}$ & 18.0 & 13.2 & 13.2 & 17.0 & 12.6 & 24.0 & 12.0 & 16.0 \\
\hline 0.052 & 3.6 & 12.6 & $\sim 6.3^{\mathrm{b}}$ & 16.5 & 9.6 & 7.8 & $?^{e}$ & 5.7 & 6.9 & 8.9 & 9.4 \\
\hline$\Delta$ & 0.1 & 0.4 & $\sim 2.2$ & 1.5 & 3.6 & 5.4 & $?$ & 6.9 & 17.1 & 3.1 & 6.6 \\
\hline \multicolumn{12}{|c|}{$C_{B}\left(\mathrm{C}_{6} \mathrm{D}_{6}\right)$} \\
\hline 1.000 & 3.6 & 11.8 & 10.0 & 16.5 & 12.8 & 13.4 & 11.4 & & 20.0 & 15.0 & 12.0 \\
\hline 0.089 & 3.5 & 10.6 & $8.3^{b}$ & 15.7 & 8.7 & $11.0^{\mathrm{b}}$ & $10.2^{b}$ & & 9.5 & 9.0 & 7.5 \\
\hline$\Delta$ & 0.1 & 1.2 & 1.7 & 0.8 & 4.1 & 2.4 & 1.2 & & 10.5 & 6.0 & 4.5 \\
\hline
\end{tabular}

aCompare Ref. 59 .

${ }^{b}$ Peaks fall on shoulder of large peak of the other isotope so there is significant uncertainty.

${ }^{\circ}$ Cannot resolve due to neighboring $\mathrm{C}_{6} \mathrm{D}_{6}$ peaks.

citon pairwise interaction energies are the same in the liquid as in the solid (the largest being $M_{\mathrm{I}, \mathrm{III}} \approx 3 \mathrm{~cm}^{-1}$ for $\mathrm{C}_{6} \mathrm{H}_{6}$ and $\sim 2 \mathrm{~cm}^{-1}$ for $\left.\mathrm{C}_{6} \mathrm{D}_{6}\right)^{35-37}$

Since the pairwise interaction parameters are very dependent on the relative positions of the molecules and since the interaction energies are essentially the same, the short range (both in time and space) structure of the liquid is probably very similar to that in the solid. In other words, the microstructure of the liquid should be nearly the same as the structure of the solid (see Fig. 6). (We notice that such pairwise interactions may change drastically with solid-solid phase transitions).

The conclusion that the microstructure of the liquid is similar to the structure of the solid is backed by the data of Narten, ${ }^{1}$ who used $\mathrm{x}$-ray diffraction and radial distribution functions, and Lowden and Chandler, ${ }^{36}$ who used the reference interaction site model as well as supporting evidence from other studies. ${ }^{12}$ Both these studies indicate that the nearest neighbors in liquid benzene, as in crystalline benzene, tend to remain perpendicular to each other.

The above does not mean that the liquid structure is "frozen," nor that the "instantaneously frozen" structure is identical to that in the solid. A variety of data indicates that things are different, and we shall limit ourselves to evidence coming from the vibrational spectra. While the crystal structure has an inversion site symmetry, ${ }^{40-42}$ and the rule of mutual exclusion is strictly obeyed ${ }^{43-51}$ in the solid, this is not quite the case in the liquid, where there are quite a few exceptions to this rule (even with ordinary instrumental sensitivity). The lowest energy $e_{2 u}$ fundamental $\left(\nu_{18}\right)$ was historically ${ }^{52}$ first observed in the Raman spectrum! On the other hand, it is about 30 times weaker than the (weak) $e_{2 s}\left(\nu_{6}\right)$ fundamental (based on our data). Also, $\nu_{12}$ and $\nu_{19}\left(e_{1 u}\right)$ were observed by early investigators. ${ }^{52}$ Similarly, six gerade bands appeared in early infrared liquid spectra. ${ }^{43,44}$ We cannot, therefore, accept the argument by Zelano and King $^{53}$ that the liquid effectively has an inversion center (they did not, however, push this point). On the other hand, based on the weakness of most bands that break the mutual exclusion rule, one can, of course, argue that the above data "confirm" the presence of an "average" $C_{i}$ site symmetry in the liquid, the exception being certain (atypical) perturbed sites (like domain boundaries or defects in a crystal). Also, from all the $D_{6 h}$ molecular symmetry elements only the center of inversion "survives" in the solid site $\left(C_{i}\right)$. Thus the inversion is the only molecular symmetry element that is at all likely to survive in the liquid. However, an interesting question is whether any of the three benzene crystal interchange symmetries ${ }^{54}$ (i.e., the three screw axes) survive. Strictly speaking, a loss of translational symmetry implies the loss of screw axes and glide planes. However, there may still be left a quasi-interchange symmetry, as in the case of dimers in doped crystals. ${ }^{55}$ That the translational symmetry is lost is not only "intuitively obvious" but can be seen from a comparison of crystal and liquid spectra near the melting point. The $A_{2 u}$ mode is the safest test case as its Davydov splitting is larger than the "thermal broadening," even close to the melting point. However, its "two pronged" spectral density in the crystal $^{37,51}$ changes into a Gaussian-like shape upon melting (Fig. 1). (The low energy shoulder due to a hot band is irrelevant to this discussion.) It is

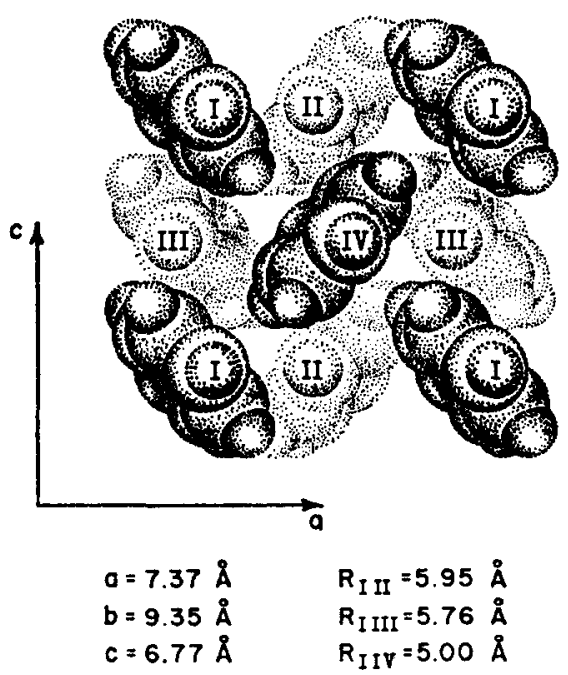

FIG. 6. Diagram of crystalline benzene viewed down the $b$ axis. The $R_{i j}$ are the center-to-center distances at $77^{\circ} \mathrm{K}$ between molecules $i$ and $j$ (from Ref. 48). 
well known ${ }^{56,57}$ that the observation of Davydov ("factor group") components in the crystal is a manifestation of selection rules due to the translational symmetry. Reports of "factor group splittings" in some molecular liquids have been discounted. ${ }^{58}$ Again we emphasize that the similarity in the energy density of states between solid and liquid benzene does not imply a similarity in spectral density (i.e., the spectroscopically observed band shape). This distinction is analogous to that found when comparing energy and spectral densities of perfect and disordered crystals. ${ }^{23}$.

That different fundamental modes have different linewidths in benzene liquid has been observed many times. ${ }^{59,18,19}$ However, whether this is caused by resonant-energy transfer or by other (i.e., translationalrotational) relaxation processes has not been clear. An empirical correlation between linewidth in the neat liquid and exciton bandwidth in the neat crystal may be suggestive, but not conclusive. Only isotopic dilution experiments appear to be conclusive in this context. It is still obvious, assuming a structural correlation between liquid and solid, that the prime vibrational band candidates for significant resonant energy transfer in the liquid are those with a significant exciton bandwidth in the solid (notwithstanding the exception discussed by Griffiths et al.). ${ }^{20}$ This, for instance, makes the 1178 $\mathrm{cm}^{-1}$ band $\left(e_{2 \xi}, \nu_{9}\right)$ the most likely candidate among the gerade fundamentals and the $992 \mathrm{~cm}^{-1}\left(a_{1 g}, \nu_{1}\right)$ about the worst (based on crystal Raman splittings ${ }^{47}$ ). One also expects similar (though not identical) behavior for corresponding $\mathrm{C}_{6} \mathrm{H}_{6}$ and $\mathrm{C}_{6} \mathrm{D}_{6}$ fundamentals. The choice of the $\nu_{1}\left(992 \mathrm{~cm}^{-1}\right)$ modes as an object of study by Griffiths et al. was obviously unfortunate from this point of view. There is no obse rvable narrowing in the $\mathrm{C}_{6} \mathrm{D}_{6}$ component and only about an $0.7 \mathrm{~cm}^{-1}$ one for $\mathrm{C}_{6} \mathrm{H}_{6}-$ too close for comfort both experimentally and theoretically (we obviously understand that in their comparative study their choice of this mode was predicated on the limitations in the novel picosecond spectroscopy method, ${ }^{20}$ and that their interpretation of the result is based on quasiresonance, i.e., intermolecular Fermi resonance ${ }^{58}$ ). We suggest that conclusive resonant transfer situations exist for those modes which (see Table I) show $\Delta \geqslant 2 \mathrm{~cm}^{-1}$ for both $\mathrm{C}_{6} \mathrm{H}_{6}$ and $\mathrm{C}_{6} \mathrm{D}_{6}$, i.e., $\nu_{8}, \nu_{9}$, $\nu_{11}, \nu_{18}$, and $\nu_{19 \cdot}$ This does not exclude a resonant vibrational energy transfer for other modes.

The nature of the resonant energy transfer interaction ("exciton interaction") is not obvious. The traditional interpretation ${ }^{60,25}$ using a transition-dipole-transitiondipole model is often suspect, and untenable for benzene (based on crystal work ${ }^{21-23}$ ). The short range repulsive part of the intermolecular potential might well play an important role in the benzene liquid (as in the crystal) ${ }^{61}$ and in other liquids. ${ }^{25}$ However, this problem is far from closed, and it may be worthwhile to collect empirically molecular pairwise resonant-energy-transfer interactions, as it has been done successfully for solids, ${ }^{23}$ and as has been true over many years for molecular force constants.

The residual width of the liquid band, obtained from isotopic dilution experiments (Table I) and ideally from extrapolation to infinite dilution (Fig. 4) is the important quantity related to nonresonant relaxation. This residual linewidth can be used $a s$ is only if one assumes that these relaxation processes are invariant to isotopic substitution $\left(\mathrm{C}_{6} \mathrm{D}_{6}\right.$ for $\left.\mathrm{C}_{6} \mathrm{H}_{6}\right)$. While this invariance is not exact, we feel that the contribution from the differential masses and moments of inertia of $\mathrm{C}_{6} \mathrm{H}_{6}$ and $\mathrm{C}_{6} \mathrm{D}_{6}$ is probably insignificant compared to the experimental uncertainty in linewidth (compare the phonon bands of $\mathrm{C}_{6} \mathrm{H}_{6}, \mathrm{C}_{6} \mathrm{D}_{6}$, and their mixtures ${ }^{62}$ ). This "residual width" of isotopically dilute solutions therefore represents a combination of both homogeneous and inhomogeneous broadening. The value for the $\nu_{11}$ band, about 3 to $4 \mathrm{~cm}^{-1}$, is in reasonable proximity to values for other modes and to values arrived at by using different methods. 14,16, 17,63-66 The values given in Table I for the most dilute concentrations $\left(0.052\right.$ for $\mathrm{C}_{8} \mathrm{H}_{8}, 0.089$ for $\mathrm{C}_{6} \mathrm{D}_{6}$ ) are rough values, owing to the preliminary nature of the data (low resolution, complications due to overlap, etc.) and are only intended as a general guide, as our emphasis in this work was on the $\nu_{11}\left(A_{2 u}\right)$ mode (note that the extrapolated, to $C_{A}=0$, linewidth obtained from Fig. 4 differs significantly from the 0.052 mole fraction linewidth in Table I). We just notice again that the contributions due to the inhomogeneity of the sites have usually been ignored. We expect the inhomogeneous broadening to be most sensitive to the nature of the excitation (mode), in close analogy to the value of the gasto-crystal (" $D$ ") shift ${ }^{48,50}$ (i.e., gas-to-liquid shift!). Another broadening effect to consider is intramolecular anharmonic interaction with the underlying vibrational density of states. This effect should be of importance only for the high frequency fundamentals (and even more so for higher combinations and overtone bands!), i.e., the $\mathrm{C}-\mathrm{H}$ stretch modes. We notice indeed that $\nu_{2}$ and $\nu_{1}$ are likely candidates for this effect.

We come back, briefly, to the interesting "sudden break" of the linewidth with concentration, exhibited in Fig. 4 and mentioned earlier. (Our data show a similar break for $C_{6} D_{6}$, at approximately the same linewidth, i.e., higher concentration.) No analogous behavior has yet been observed in solids (to our knowledge). However, one can predict it, qualitatively, if one considers a variation of the homogeneous broadening effect (exciton-phonon coupling) with concentration, i. e. , excitation bandwidth. It is known $\mathrm{n}^{67-69}$ that homogeneous broadening involves a different mechanism for localized excitons (dilute solid solutions) than for the extended excitons (neat solids). The transition from the latter case to the first one may happen over a small concentration range and cause the above anomaly. Alternatively, a detailed study of the "intermediate coupling case," involving exciton-phonon coupling and its variation with exciton bandwidth (not assuming a change in the coupling itself), may also give such a result. This phenomenon deserves further study-both experimentally and theoretically, in solids as well as liquids.

Finally, we notice that the $40 \mathrm{~cm}^{-1}$ total width of the liquid exciton band implies an overall resonance interaction of roughly $\sum_{j} M_{i j}=20 \mathrm{~cm}^{-1}$ of a molecule $i$ with all its neighbors $j$, i. e., it looses ${ }^{71}$ its vibrational excitation to one of them in about ${ }^{72} 0.3$ psec. Assuming the 
largest nearest neighbor pairwise molecular interaction to be the same as in the solid, one gets a $3 \mathrm{~cm}^{-1}$ interaction or an "incoherent jump time" of about ${ }^{73} 2$ psec. On the other hand, the "residual width" (at isotopic dilution) of about $3-4 \mathrm{~cm}^{-1}$, which is due to homogeneous and/or inhomogeneous broadening, gives a lower lim it of reorientational and/or translational relaxation time of about ${ }^{73} 2$ psec., in reasonable agreement with literature values (see above). $14,16,17,63-66$

\section{CONCLUSIONS}

From our data it can be concluded that the width of the $A_{2 u}$ exciton band in liquid benzene is about $40 \mathrm{~cm}^{-1}$, which is comparable to that of the solid. Thus the interaction energies are nearly the same and the microstructure of the liquid must be similar to the structure of the solid. ${ }^{1}$ The much smaller residual linewidth of the isotopically infinitely dilute molecules $\left(3-4 \mathrm{~cm}^{-1}\right.$, by extrapolation) is probably the quantity involved in the nonresonant relaxation processes, unless it is due to inhomogeneous broadening. In contrast, the $40 \mathrm{~cm}^{-1}$ exciton bandwidth is a measure of the fastest "relaxation" channel, i.e., the resonant energy transfer. The corresponding nearest neighbor pairwise molecular interaction is about $3 \mathrm{~cm}^{-1}$. The vibrational $\left(A_{2 u}\right)$ resonant transfer rate is thus about 1 psec.

\section{ACKNOWLEDGMENT}

We wish to thank Dr. H. - K. Hong for some preliminary experiments.

*Supported by NSF Grant DMR75-07832 A01 and NIH Grant NS08116-08.

'Present address: Dept. of Chemistry, Harvard University, Cam bridge, MA 02138.

${ }^{1}$ A. H. Narten, J. Chem. Phys. 48, 1630 (1968).

${ }^{2}$ s. Katzoff, J. Chem. Phys. 2, 841 (1934).

${ }^{3}$ W. C. Pierce, J. Chem. Phys. 5, 717 (1973).

${ }^{4}$ P. H. Bell and W. P. Davey, J. Chem. Phys. 9, 441 (1941).

${ }^{5} A$. Van Itterbuk and A. de Bock, Nature (London) 162, 611 (1948).

${ }^{6} \mathrm{G}$. Dezelie, J. Chem. Phys. 45, 185 (1966).

${ }^{7}$ D. J. Coumon, E. L. Mackor, and J. Himmans, Trans. Faraday Soc. 60, 2244 (1964).

${ }^{8}$ R. L. Schmidt, J. Colloid Interface Sci. 27, 516 (1968).

${ }^{9} \mathrm{M}$. S. Malmberg and E. R. Lippincott, J. Colloid Interface Sci. 27, 591 (1968).

${ }^{10}$ A. I. Sokolovskaya, Tr. Fiz. Inst. Acad. Nauk SSSR 27, 63, (1969).

${ }^{11}$ L. A. Blatz, J. Chem. Phys. 47, 841 (1967).

${ }^{12}$ R. L. Schmidt and J. H. Goldstein, J. Chem. Phys. 59, 1494 (1969).

${ }^{13}$ S. K. Gorg, J. E. Bertie, H. Kilp, and C. P. Smyth, J. Chem. Phys. 49, 2551 (1968).

${ }^{14}$ T. E. Bull and J. Jonas, J. Chem. Phys. 52, 4553 (1970).

${ }^{15}$ D. E. Woessner, J. Chem. Phys. 40, 2341 (1964); D. E. Woessner and B. S. Snowden, J. Chem. Phys. 52, 1621 (1970).

${ }^{16} \mathrm{~J}$. F. Dill, T. A. Litovitz, and J. A. Bucaro, J. Chem. Phys. 62, 3839 (1975).

${ }^{17} \mathrm{~A}$. Ben-Reuven and N. D. Gershon, J. Chem. Phys. 51, 893 (1969).

${ }^{18}$ F. J. Bartoli and T. A. Litovitz, J. Chem. Phys. 56, 404 (1972).

${ }^{19}$ F. J. Bartoli and T. A. Litovitz, J. Chem. Phys. 56, 413 (1972).
${ }^{20}$ J. E. Griffiths, M. Clerc, and P. M. Rentzepis, J. Chem. Phys. 60, 3824 (1974); but see A. Laubereau and W. Kaiser, Ann. Rev. Phys. Chem., 26, 83 (1975).

${ }^{21}$ R. Kopelman, Rec. Chem. Progr. 31, 211 (1970).

${ }^{22}$ R. Kopelman, J. Chem. Phys, 47, 3227 (1967).

${ }^{23}$ R. Kopelman, in Excited States edited by E. C. Lim, (Academic, New York, 1975), Vol. II.

${ }^{24}$ D. J. Lockwood, J. Scott, W. L. Elsdon, and D. E. Irish,

J. Raman Spectra 2, 593 (1974).

${ }^{25} \mathrm{~T}$. Tokuhiro and W. G. Rothschild, J. Chem. Phys, 62, 2150 (1975).

${ }^{26}$ S. A. Rice and J. Jortner, J. Chem. Phys. 44, 4470 (1966).

${ }^{27} \mathrm{G}$. Nicolis and S. A. Rice, J. Chem. Phys. 46, 4445 (1967).

${ }^{28} \mathrm{~W}$. L. Greer, S. A. Rice, and G. Morris, J. Chem. Phys. $52,5622(1970)$.

${ }^{29} \mathrm{~B}$. Raz and J. Jortner, J. Chem. Phys. 49, 3318 (1968); Proc. R. Soc. London. Ser. A 317, 113 (1970).

${ }^{30}$ C. J. Eckhardt and L. F. Nichols, Phys. Rev. Lett. 29, 1221 (1972).

${ }^{31}$ W. P. Helman, J. Chem. Phys. 51, 354 (1969).

${ }^{32}$ P. Holzman and R. C. Jarnagin, J. Chem. Phys. 51, 2251 (1969).

${ }^{33}$ H. Baessler, J. Chem. Phys. 49, 5198 (1968), and references therein.

${ }^{34} \mathrm{~J}$. C. Laufer and R. Kopelman, J. Chem. Phys. 53, 3674 (1970).

${ }^{35} \mathrm{~J}$. C. Laufer and R. Kopelman, J. Chem. Phys. 57, 3202 (1972).

${ }^{36} \mathrm{~J}$. Hoshen and R. Kopelman, Phys. Status Solidi B 81, 0000 (1977).

${ }^{37}$ R. LeSar and R. Kopelman (unpublished).

${ }^{38}$ L. J. Lowden and D. Chandler, J. Chem. Phys. 61, 5228 (1974).

${ }^{39}$ H-K. Hong and R. Kopelman, J. Chem. Phys. 55, 5380 (1971).

${ }^{40}$ E. G. Cox, Rev. Mod. Phys. 10, 159 (1958).

${ }^{41}$ E. G. Cox, D. W. Cruickshank, and J. A. S. Smith, Proc. R. Soc. London Ser, A 217, 1 (1958).

${ }^{42}$ G. E. Bacoil, N. A. Curry, and S. A. Wilson, Proc. R. Soc. London Ser. A 279, 98 (1969).

${ }^{43}$ R. S. Halford and O. A. Schaeffer, J. Chem. Phys. 14, 141 (1946).

${ }^{44}$ R. Mair and D. F. Hornig, J. Chem. Phys. 17, 1236 (1949).

${ }^{45}$ A. Fruhling, Ann. Phys. (Paris) 6, 26 (1951).

${ }^{46} \mathrm{~S}$. Zwerdling and R. S. Halford, J. Chem. Phys. 23, 2221 (1955).

${ }^{47}$ A. R. Gee and G. W. Robinson, J. Chem. Phys. 46, 4847 (1967).

${ }^{48}$ E. R. Bernstein, S. D. Colson, R. Kopelman, and G. W. Robinson, J. Chem. Phys. 48, 5596 (1968).

${ }^{49}$ S. D. Colson, R. Kopelman, and G. W. Robinson, J. Chem. Phys. 47, 27, 5462 (1967).

${ }^{50}$ E. R. Bernstein, J. Chem. Phys, 50, 4842 (1969).

${ }^{51}$ E. R. Bernstein and G. W. Robinson, J. Chem. Phys. 49, 4962 (1968).

${ }^{52} \mathrm{G}$. Herzberg, Infrared and Raman Spectra of Polyatomic Molecular (Van Nostrand, Princeton, NJ, 1945), p. 364.

${ }^{53}$ A. J. Zelano and W. T. King, J. Chem. Phys. 53, 4444 (1970).

${ }^{54}$ R. Kopelman, J. Chem. Phys. 47, 2631 (1967).

${ }^{55} \mathrm{H}-\mathrm{K}$. Hong and R. Kopelman, J. Chem. Phys. 57, $3888(1972)$.

${ }^{5} \mathrm{H}$. Winston and R. S. Halford, J. Chem. Phys. 17, 607 (1949); D. F. Hornig, J. Chem. Phys. 16, 1063 (1948).

${ }^{57}$ A. S. Davydov, Theory of Molecular Excitons (McGraw-Hill, New York, 1962).

${ }^{58}$ R. Kopelman, J. Chem. Phys. 44, 3547 (1966).

${ }^{59}$ J. D. Masso, Y. D. Harker, and D. F. Edwards, J. Chem. Phys. 50, 5420 (1969).

${ }^{60}$ D. A. Dows, Physics and Chemistry of the Organic Solid State, edited by D. Fox, M. M. Labes, and A. Weissberger (Interscience, New York, 1963), Vol. I, p. 609.

${ }^{61}$ G. Taddei, H. Bonadeo, M. P. Marzocchi, and S. Califano 
J. Chem. Phys. 58, 966 (1973).

${ }^{62} \mathrm{H}-\mathrm{K}$. Hong and R. Kopelman, J. Chem. Phys. 58, 384 (1973).

${ }^{63} \mathrm{D}$. R. Bauer, G. R. Alms, J. I. Brauman, and R. Pecora, J. Chem. Phys. 61, 2255 (1974).

${ }^{64}$ G. R. Alms, D. R. Bauer, J. I. Brauman, and R. Pecora,

J. Chem. Phys. 58, 5570 (1973).

${ }^{65} \mathrm{~K}$. T. Gillen and J. E. Griffiths, Chem. Phys. Lett. 17, 359 (1972).

${ }^{66}$ R. Kesfeld, B. B. Gross, and W. Dietrick, Z. Naturforsch. Teil A 25, 40 (1970).

${ }^{67}$ P. N. Prasad, Ph.D. thesis, Univ. of Pennsylvania, 1971.

${ }^{68}$ R. M. Hochstrasser and P. N. Prasad, J. Chem. Phys. 57,
5409 (1972).

${ }^{69}$ R. M. Hochstrasser and P. N. Prasad, Excited States $I$. (Academic, New York, 1973).

${ }^{70}$ J. Hoshen and R. Kopelman, J. Math. Phys. 17, 2067 (1976). ${ }^{71} \mathrm{P}$. Argyrakis and R. Kopelman (unpublished).

${ }^{72}$ Using: $t \approx\left(2 \pi \Sigma, M_{t}\right)^{-1}$. The $2 \pi$ factor is dubious. ${ }^{73}$

${ }^{73}$ Using: $t \approx(2 \pi M)^{-1}$. The factor $2 \pi$ is used here and above to conform with most of the literature on liquids. However we have not used it elsewhere. ${ }^{71}$ For a recent discussion see Ref. 74 .

${ }^{74}$ G. W. Robinson and J. O. Berg, Can. J. Phys. 53, 2068 (1975). 